\title{
Segregación socioespacial urbana. El ejemplo de Tijuana
}

\section{Tito Alegría*}

Este trabajo tiene como objetivo explorar de manera conceptual y empírica la relativa homogeneidad social dentro de las zonas residenciales y la heterogeneidad entre ellas, para lo cual hacemos una discusión conceptual y un ejercicio estadístico.

En términos conceptuales, se discute la naturaleza de la segregación socioespacial acudiendo al concepto de distinción-diferenciación (desarrollado por $P$. Bordieu), abordándose también la relación entre la segregación social en el espacio urbano y los niveles de ingreso (el indicador más usual) y conocimiento (en el sentido dado por $N$. Thrift).

Al respecto se propone que la diferenciación social se materializa en la praxis de la disputa por los recursos urbanos entre los residentes de la ciudad. Esta disputa (conflictiva o no) ocurre a través de dos dimensiones sociales complementarias, el mercado y los mecanismos institucionales. La capacidad de pago y el conocimiento de los residentes determinan sus ventajas en ambas dimensiones sociales. Las personas al identificarse con otros (en el sentido de reconocerse a símismos en la praxis del otro) que tienen similares atributos de pago y conocimiento, van diferenciándose de los que tienen otros atributos. Las personas ubicadas en la cúspide de la pirámide social pueden diferenciarse con mayor facilidad, y la capacidad de distinción decae con el ingreso, bajando por la pirámide social.

Debido a que muchos de los recursos urbanos son inmóviles, la disputa por ellos incorpora la dimensión espacial en términos de cercanía-lejanía. De este modo, la diferenciación social se materializa como segregación espacial. La segregación de las personas con mayores ventajas de pago y (o) conocimiento se convierte en autosegregación.

El objetivo empírico es probar la relación estadística entre segregación espacial e ingreso y conocimiento. Los datos que se usan son del censo de población 1990 a escala de AGEB.

Este trabajo tiene como objetivo explorar las variaciones del grado de homogeneidad social entre las diferentes zonas de una ciudad, y es parte de uno mayor sobre la estructura interna de las ciudades de la frontera de México con Estados Unidos. Específicamente, forma parte de la búsqueda de explicaciones sobre la localización de grupos sociales y actividades económicas respecto a los recursos urbanos. Tomamos en cuenta que los integrantes de las entidades (grupos o sectores) sociales y económicas compiten por

\footnotetext{
* El Colegio de la Frontera Norte.
} 
los lugares que tienen ventajas de localización por su accesibilidad y compatibilidad con los recursos de infraestructura y equipamiento urbanos. La competencia ocurre entre entidades instrumentadas de manera desigual -que constituyen la demanda sobre los sitios- actuando sobre mercados inmobiliarios de competencia imperfecta que se expresan en diversas funciones de precios inmobiliarios.

Enfocamos una parte de las entidades demandantes, los grupos sociales. El despliegue de éstos sobre el territorio de la ciudad se verifica con ciertos patrones espaciales de mezcla y localización. La mezcla nos informa del predominio cuantitativo de algún grupo social en cada zona. La existencia de este predominio se manifiesta como homogeneidad social del lugar, y la ausencia de aquél, como heterogeneidad. Este ensayo trata más sobre los patrones espaciales de mezcla social que de aquéllos de localización residencial. Para ello, presentamos una discusión sobre la conceptualización de la segregación y sus determinantes, y a continuación un modelo estadístico que examina la pertinencia de esos determinantes.

\section{Sobre la conceptualización de la segregación}

En los estudios urbanos se ha intentado explicar la segregación socioespacial como asociada a los patrones de localización intraurbana de los grupos sociales, sea con enfoques sociológicos o económicos. En algunos casos, la localización aparece de manera explícita como momento teórico que relaciona los mecanismos económicos y la segregación, como acontece con los enfoques microeconómicos. En otros casos, los patrones de localización son resultado implícito de la segregación socioespacial, como lo presentan los enfoques de la marginalidad. Lo que es común a esos enfoques es no haber establecido la relación teórica entre segregación y localización, impidiéndose así una conceptualización de la segregación con un estatus diferenciado de la localización.

El enfoque de marginalidad urbana interpretó la segregación como una condición de exclusión: de las ventajas del sector moderno en la vertiente funcionalista (Germani, 1988), y del sector económico-social-político dominante y formal en la versión dependentista (Quijano, 1988). En ambas versiones la segregación social era conceptualizada como la marginalidad social, desplegándose en el territorio urbano, en los centros y en los bordes. $\mathrm{Al}$ llamar a estos últimos "cinturones de miseria" se hacía idéntica, de manera implícita, la situación de marginalidad social con la 
localización marginal en el espacio urbano. En la misma línea de pensamiento, las personas participantes de los sectores modernos no eran marginales y por ende no estaban segregadas. En este enfoque, las grandes concentraciones de gente pobre localizadas en las periferias de la ciudad se entendían como resultado de la falta de participación en los sectores dinámicos y (o) dominantes de la economía. De esta manera, la localización, en tanto un sistema de asignación de usos residenciales del suelo, no tuvo un estatus autónomo respecto a los mecanismos de segregación. La topología de las personas dentro de la estructura económica determinaba la ubicación social y ambas, la localización espacial. No había espacio para la modificación de la estructura debido a las iniciativas de los agentes basadas en sus propias y multidimensionales características y preferencias de localización. El cambio del espacio urbano no se regiría por una disputa por las localizaciones sino por el acceso a los sectores privilegiados de la estructura social y económica, es decir, los no marginales.

En el enfoque neoclásico del análisis urbano -desarrollado para ciudades de países con gran capacidad de acumulación del capital- la segregación es producto de condiciones sociales exógenas a, y operando sobre, los mecanismos de mercado que determinan la localización residencial (Alonso, 1988). En un mercado de competencia perfecta, la gente pobre debido a su limitada capacidad de gasto en espacio de vivienda tiene que habitar en unidades pequeñas, en zonas de alta densidad poblacional alrededor de los centros urbanos; las personas de mayores ingresos que pueden gastar más en vivienda, habitan en las zonas menos densas, en las periferias de la ciudad. La distribución (matemáticamente) continua de la población según niveles de ingreso asigna teóricamente una distribución espacial continua de la población según el nivel de ingreso ascendente desde el centro de la ciudad hacia la periferia. Como la distribución es continua no existe segregación en tanto bolsones urbanos homogéneos de asentamientos diferenciables de los demás. Aunque este modelo teórico no abarca la segregación, ésta existe en la realidad. Para explicarla -intentando explicar sólo la segregación de los más pobres-, consideran condiciones exógenas como la raza, la procedencia migratoria o el nivel de educación de los habitantes. Estas condiciones actúan como segmentos sociales o como determinantes de ingresos similares, que se materializan en el espacio como necesidad de contigüidad o asignación de similar localización. Como es notable, este enfoque falla al considerar sólo los determinantes económicos para reproducir teóricamente la lógica de la localización residencial; pero además, es incapaz de explicar por sí la segregación 
socioespacial de las ciudades. Las consecuencias espaciales de este enfoque tampoco expresan la realidad de las ciudades latinoamericanas debido a que los pobres habitan principalmente las periferias ocupando zonas de baja densidad.

En el enfoque neomarxista predominante, la segregación entre grupos sociales es el resultado del ejercicio del poder de la clase dominante sobre los mecanismos del mercado inmobiliario (Harvey) para obtener ganancias y asegurar la reproducción del sistema. La dinámica de la urbanización responde a la lógica del sector financiero y del gobierno; esa dinámica es mediada por los especuladores-promotores y especuladores-terratenientes en la búsqueda de las rentas del suelo. La extracción de la renta de la tierra urbana -que ocurre dentro del proceso de consumo más no del proceso de producción- es la fuente de los problemas y conflictos en comunidades o barrios. En el desenvolvimiento de los conflictos, los habitantes de un barrio van adquiriendo conciencia de sí como una comunidad diferenciada de las demás. De este modo, la renta del suelo produce una diferenciación-segregación para sí tanto como en sí. En otro nivel de análisis, al producir nuevos modos de consumo y nuevas carencias y necesidades, el proceso de urbanización genera, de manera concomitante, nuevos grupos por distribución del ingreso o clases de consumo. Estos segmentos sociales así conformados, de manera multidimensional, pueden cristalizar en comunidades diferenciadas dentro de la estructura urbana. La segregación es el resultado espacial de la cristalización de diversas comunidades. En cambio, las localizaciones de estas comunidades responden à mecanismos de mercado inmobiliario (en la esfera del consumo) modulados por la lógica del sector financiero y el gobierno e impulsados por el interés del capital inmobiliario ante la diversidad de carencias y necesidades de las comunidades. Los individuos pueden saltar de un grupo social a otro o entre comunidades, pero su acción no modifica el mecanismo de generación de las comunidades y de la segregación. En este enfoque se vislumbra la separación conceptual de segregación y localización, sin embargo tal separación no es desarrollada.

Considerar la segregación como la distancia económica o social entre un grupo de personas y los recursos urbanos -como ya vimos que algunos enfoques proponen- lleva implícita la identidad espacial entre segregación y localización. Esta identidad es un error conceptual pues impide la explicación de la localización diferente de grupos similares (por ejemplo los grupos de ingreso) y la localización similar de grupos diferentes (por ejemplo los grupos por estatus profesional). Para resolver este problema pro- 
ponemos una conceptualización diferente de la segregación, una que esté definida como la separación o adyacencia espacial entre grupos sociales y no de los grupos respecto a cualquier otra entidad urbana.

El mecanismo económico de la segregación

La mayor o menor homogeneidad social de una zona de la ciudad es el resultado, primero, del enfrentamiento de segmentos de los diferentes grupos sociales por conseguir una localización (expresando la demanda), o un inmueble, con particulares características propias y de entorno (expresando la oferta); y segundo, de las mutuas modificaciones entre oferta y demanda que ocurren en la dimensión temporal y que se expresan en precios inmobiliarios ${ }^{1} \mathrm{di}-$ ferenciados entre las zonas de la ciudad. Mayor homogeneidad social dentro de cada una de las zonas de la ciudad significa mayor heterogeneidad entre zonas, y ambas características indican mayor segregación espacial entre grupos sociales.

A la competencia por la localización, cada segmento social lleva diferentes recursos y diferentes objetivos para y por los que competir. Entre recursos y objetivos hay nexos subyacentes que los hacen formar parte de un mismo proceso racional de adecuamiento de medios a fines. Aunque ello no significa que los fines sean necesariamente determinados de manera racional ni que los medios sirvan y se hayan obtenido para conseguir una única localización. Debido a que medios y fines -o recursos y objetivos-conforman una unidad en la acción individual o grupal, las dimensiones subyacentes comunes entre ellos no pueden ser sino sus determinantes. Conceptualmente, estas dimensiones son las que le dan unidad a medios y fines, sustentando y modulando de manera individual la lógica de ambos. De acuerdo con el modo en que pueden modificar recursos y objetivos, a esas dimensiones-determinantes las podemos agrupar de varias maneras, pero debido a las condiciones de la competencia por la localización, es mejor agruparlas en económicas y de conocimiento de su sociedad. En consecuencia, el instrumental con que cada individuo de cada grupo social entra a la competencia dependerá del nivel y tipo de recursos económicos y de conocimiento, y sus objetivos de localización en el espacio urbano dependerán también de ambos tipos de características.

${ }^{1}$ Los precios del suelo son el resultado, en la dimensión temporal, de la competencia entre todas las entidades urbanas incluyendo las sociales y las económicas que se manifiestan en el espacio como usos del suelo diferenciados. 
Estas características nos permiten clasificar a los grupos sociales en $M$, grupos de acuerdo con sus recursos económicos, y en $N$. en relación con aquellos de conocimiento. Cada clasificación corresponde a un campo, el cual es definido por Bourdieu como un espacio (no territorial) determinado, donde se despliegan las relaciones de fuerza entre los agentes o instituciones (entidades) propias al campo, y su estructura es la distribución entre las entidades, del capital específico al campo (por ejemplo el económico o el de conocimiento) acumulado en luchas anteriores y que orientan las estrategias ulteriores (Bourdieu, 1990: 136). Cada campo es la arena en que se despliega la acción y lucha de los grupos sociales. Hay tantos campos como tipo de capitales por qué luchar, y unos inciden más que otros en la determinación de las prácticas cotidianas. En consecuencia, un individuo o grupo o institución pertenece a la vez a muchos campos. Dentro de cada campo o clasificación, hay una tendencia a la diferenciación social entre clases o grupos que se plasma también en términos espaciales, es decir, como segregación residencial.

En realidad, la segregación está asociada a la localización aunque constituyan dos procesos diferentes. Muchos campos inciden en las decisiones de localización residencial, por ejemplo el simbólico y el político; pero los más influyentes son el económico y el de conocimiento. Tomando en cuenta sólo estos dos últimos, la cantidad de grupos sociales sería $N x M$. Si no hubiera restricciones o imperfecciones por el lado de la oferta de sitios habría $M x N$ grupos sociales, cada uno ocupando una zona relativamente homogénea, sustentando el proceso de segregación residencial entre grupos.

Sin embargo, cada uno de esos $N x M$ grupos participa de manera diferente en las dos formas de la arena de disputa económica por los recursos urbanos localizados, que son la de mercado y la institucional. La primera es un conjunto de mercados generalmente distorsionados por imperfecciones referidas a monopolio, segmentación e indivisibilidad. Ejemplo de ellos son los mercados de bienes inmuebles y públicos. La segunda forma, la institucional, abarca el conjunto de medios fuera del mercado pero relacionados con él, a través de los cuales se obtienen ventajas en precio, localización o simplemente acceso a un mercado particular. Evidencia de este tipo, son los medios sindicales, políticos y las instituciones civiles como asociaciones de empresarios, de invasores de terrenos, de colegios profesionales, etcétera. Los grupos çn mayoìes recursos económicos tendrán la mayor ventaja en la competencia por la localización. Sin embargo, cada clase económica $M$ está dividida en $N$ clases según grados de conocimien- 
to, de manera que en un mismo nivel económico, las diferencias en el tipo y nivel de conocimiento propiciarán diferentes localizaciones óptimas. De manera recíproca, a un mismo tipo de conocimiento, las diferencias en los recursos económicos generarán diferentes localizaciones. ${ }^{2}$

Los individuos de cada grupo social intentarán lograr una localización óptima cuando experimenten una discordancia entre sus características y las del sitio donde está su hogar. Cuando se esté experimentando tal discordancia ellos pasarán a un estado de cambio potencial de localización que se desplegará cuando sepan de alguna localización que corresponda al nuevo estado de sus recursos económicos y de conocimiento. En este proceso debieran irse conformando áreas con relativa homogeneidad, que al tener similares características de sitio, tendrían personas con similares características económicas y de conocimiento. Sin embargo, la localización óptima para cada nivel económico y de conocimiento no se puede alcanzar debido a que el cambio del stock de sitios disponibles corre a diferente velocidad que el cambio de las características económicas y de conocimiento. El stock de sitios disponibles está modulado por las imperfecciones estructurales del mercado inmobiliario, las cuales son un gran freno para la homogenización de una zona urbanizada. En el largo niazo, se añade como modificador de la segregación (retardándola o acelerándola) el modo en que ocurre la expansión física de la ciudad en relación con la velocidad y la forma de ocupación de nuevos sitios para vivienda. Direcciones geográficas con rápido crecimiento físico generalmente han añadido -de manera discontinua-grandes porciones de terreno a la ciudad, ocupado por personas socialmente menos heterogéneas, a través tanto de invasiones de gente pobre como de fraccionamientos para grupos de ingreso medio y alto. En contraste, direcciones geográficas que han crecido lentamente añadieron cada vez pequeñas porciones de terreno no pudiendo por ello tener en las zonas que componen niveles altos de homogeneidad social.

2 Las teorías marginalistas de uso del suelo asignan, de manera no realista, una misma localización a las familias con ingresos similares, en razón del supuesto según el cual, los pobladores urbanos tienen gustos y preferencias similares, desconociendo las diferencias de conocimiento que surgen tanto de las diferencias de ingreso como de aquellas que emergen de otros campos. 


\section{El mecanismo social de la segregación}

La segregación social se manifiesta en el espacio como segregación residencial. Conceptualmente, es el proceso de diferenciación socioespacial urbana que comprende dos dimensiones complementarias y necesarias: similares características económicas y de conocimiento dentro de una zona, y diferentes características entre zonas. Así, la segregación sería nula en el caso de que en todas las zonas hubieran habitantes de todo tipo.

Vista por el lado de la demanda, es decir de la distribución espacial de los diferentes consumos, la segregación es una cara de las múltiples que tiene la distinción social. Pero la distinción no es necesariamente el objetivo de los consumos, sino que surge objetivamente por el hecho individual de consumir bienes diferentes. Como hay algunas personas que compran bienes parecidos o iguales, se conforman grupos de personas con consumos similares. Estos grupos afirman su legitimidad en la defensa de la propia elección de consumo ante los demás grupos al incorporarse en el campo de la disputa del poder simbólico. De esta manera constituyen grupos con cierta cohesión alrededor de los símbolos, en el que por el propio desenvolvimiento de la disputa por el poder simbólico, cada grupo se diferencia a sí mismo de los demás en la ciudad. La localización de la vivienda es la principal diferencia entre los bienes residenciales individuales; pero su consumo individual y la atribución de valor a ese consumo, se realiza con total falta de independencia del consumo de los vecinos. De manera que las diferencias y disputas entre vecinos (generalmente menores) no ponen en cuestión las bases que conforman cada grupo, y se soslayan ante la diferenciación ejercida en el campo simbólico frente a otros barrios y zonas de la ciudad. La segregación se alimenta de esta disputa en el campo simbólico.

Considerar que la segregación socioespacial se genera en este último campo sería suponer que los consumos se realizan con el objetivo de distinguirse. Sin embargo, como ha mostrado Bourdieu, los consumos tienen más que ver con el habitus, sistema de disposiciones adquiridas por medio del aprendizaje implícito o explícito; funciona como un sistema de esquemas generadores de estrategias que pueden estar objetivamente conformes con los intereses objetivos de sus autores sin haber sido concebidas expresamente con este fin (Bourdieu, 1990: 141). La distinción es sólo una entre múltiples estrategias cuyos objetivos pueden estar implícitos en las elecciones de consumo pero no haber sido concebidos como finalidad. Las elecciones similares de consumo residencial 
están de algún modo programadas para los individuos que tienen habitus similares, y se van reprogramando en el proceso permanente de reconstitución del grupo de referencia como producto de la continua y mutua modificación entre el habitus y el consumo del bien residencial.

Un habitus particular se constituye sobre determinados recursos (o capital) económicos del individuo. Para diferente capital económico habrá diferentes modalidades posibles de habitus. Este es el momento teórico en que el capital de conocimiento y el capital económico se conjugan para hacer una elección residencial. El caudal de conocimiento y el económico imponen los límites al rango de posibilidades de elección. El mecanismo de selección específica dentro de ese rango lo constituye la obtención de la máxima rentabilidad dentro del campo económico (es decir, el menor precio entre viviendas similares en otras características) y mayor adecuación con su habitus (es decir, el sitio que facilita la reproducción de la mayor cantidad de prácticas propias al segmento social de conocimiento al que se siente pertenecer). De esta manera, la segregación no es un objetivo implícito en las elecciones de localización, pero es su resultado y se corresponde con los mecanismos económicos y con la defensa del habitus propio.

\section{Conocimiento}

Dentro de la corriente neoclásica, conocimiento en las decisiones de localización es el saber sobre las posibilidades residenciales ofertadas, sus características y su precio. Debido a condiciones estructurales del mercado residencial es imposible conocer todas las posibilidades de elección antes de tomar la mejor decisión (es decir, la que permita la mayor utilidad o satisfacción). Sin embargo, aunque incompleto y a veces distorsionado, hay un saber sobre los mecanismos de acceso a la vivienda que se constituye dentro del campo del conocimiento social (en el sentido de Bourdieu) y bajo sus reglas.

Cada persona tiene un particular nivel y tipo de conocimiento de la relación entre la acción de un individuo y la estructura social o de mercado que la modula. Este saber está limitado por la experiencia en la producción de prácticas y su continua reinterpretación a la luz de los eventos subsecuentes (Thrift, 1985: 368). Estos límites están constituidos por: i) la singular experiencia biográfica de practicar un particular sistema social; ii) porque todo conocimiento es el resultado de un particular habitus usado para generar prácticas y sus interpretaciones; iii) por el hecho de que 
todas las prácticas están contenidas en determinados tiempos y espacios, y iv) porque el conocimiento se puede comunicar sólo parcialmente debido a las restricciones que imponen los tres límites anteriores.

Cada sociedad tiene un stock de conocimiento conformado por los saberes particulares de sus integrantes. Sin embargo, está distribuido de manera desigual dependiendo de las numerosas dimensiones que estructuran a los grupos sociales, tales como diferencias biológicas (género, raza, etcétera), clase (posición en el sistema productivo), ingreso (todas las rentas personales), etcétera. Cada grupo social tiene un nivel de conocimiento de su medio dependiendo de su capacidad para obtenerlo.

Cada grupo social o cada individuo tiene cuatro tipos de conocimiento (Thrift, 1985: 372) que incorpora al stock social del conocimiento: i) Conocimiento inconsciente, que está basado sobre las estructuras objetivas de acción, generadas en prácticas pasadas, y que son incorporadas al habitus. ii) Conocimiento práctico, que es un tipo de conocimiento informal aprendido de la experiencia de "ver y hacer" en contextos muy particulares, en la relación con otras personas, y acotada en tiempo y espacio. iii) Conocimiento empírico, cuyo stock es construido como resultado de un proceso general de racionalización del conocimiento bajo dos modalidades, dando explicaciones racionales y organizando el conocimiento en una forma sistemática. iv) Conocimiento filosófico, basado en el intento de unificar los diferentes cuerpos de conocimiento en uno solo.

Como ya vimos, la segregación socioespacial está compuesta por la relativa homogeneidad/heterogeneidad de cada una de las zonas de la ciudad. Tanto el conocimiento práctico como el inconsciente están acotados por el espacio, principalmente el residencial. Estos conocimientos se van acumulando y reproduciendo con el tiempo en plazos largos, generando límites espaciales definibles y modificaciones lentas al habitus. Por ello, las personas y grupos que más tiempo hayan habitado la ciudad tendrán más de estos tipos de conocimientos y se localizarán en zonas con relativa homogeneidad. Además, estas personas y grupos en ciudades con poca proporción de nativos, como sucede en la frontera, tenderán a concentrarse en pocas áreas. Si las personas y grupos añaden a esos dos tipos de conocimientos el empírico, entonces se ubicarán en zonas más heterogéneas pero concentradas alrededor de los mejores recursos urbanos. El conocimiento empírico tiene menos limitantes espaciales y más temporales, respecto a los dos primeros tipos de conocimientos: se desarrolla en esferas institucionales (escuelas, sindicatos, partidos políticos, cámaras, 
colegios, clubs, gobierno, etc.) con poca dependencia de las localizaciones residenciales, y este saber, cambiante, se adquiere sólo en el caso temporalmente determinado de la participación efectiva dentro de tales instituciones. Las personas y grupos con menos conocimiento inconsciente, práctico y empírico serán marginadas a zonas con menores ventajas de localización y con tendencia a la homogeneidad. Aquellos que detenten mayor cantidad de conocimiento empírico (y poca de inconsciente y práctico) tendrán la posibilidad de dispersarse en busca de ventajas inmóviles (de sitio y vivienda) y ejerciendo ventajas móviles (financieras o de acceso a un submercado), y por ello participarán de zonas con relativa heterogeneidad. El esquema siguiente presenta la relación entre conocimiento y localización.

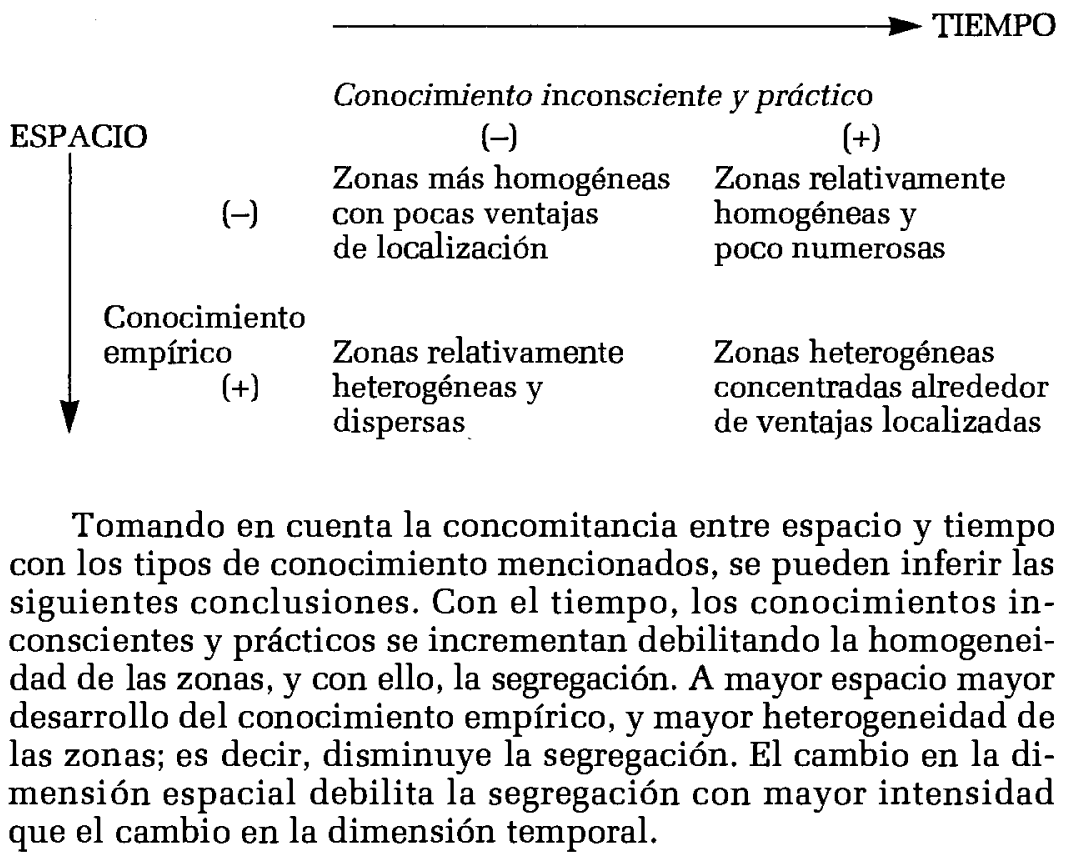

\section{Ingresos}

Las personas y grupos sociales en la ciudad desarrollan una competencia entre sí en diversos campos (económico, simbólico, profesional, etc.), participando con diferentes tipos de capitales correspondientes a cada campo y con el objeto de plusvalorarlos. 
Los campos están arreglados jerárquicamente entre sí, de manera que los mutuos condicionamientos son desiguales en intensidad y sentido. Debido a que la segregación se produce en la elección residencial, consideramos como campo económico a la esfera del consumo. Este campo ocupa un lugar privilegiado en la jerarquía, debido a que organiza las posibilidades de competir con ventaja (o sin ella) en los otros campos, y a que discrimina diversas disposiciones con las cuales los habitus se producen y se reproducen de manera confirmatoria-transformada y legitimadora.

La capacidad de consumo está en la accesibilidad al ingreso. A igual grado de conocimiento, las variaciones del ingreso familiar distribuirán socialmente los consumos residenciales. En un mercado de competencia perfecta, el despliegue espacial de esta diferenciación social del consumo dependería de la distribución territorial de los precios. Pero por las imperfecciones estructurales del mercado residencial, los precios nos dan sólo una idea de la distribución espacial de los grupos de ingreso en un mismo nivel de conocimiento.

Si dividimos a la sociedad en grupos de ingreso, su distribución dentro de las zonas urbanas nos indicaría la magnitud de la homogeneidad o heterogeneidad de cada una de ellas, en otras palabras, la magnitud de uno de los dos componentes de la segregación socioespacial. El segundo componente, es decir, la heterogeneidad entre zonas, se obtendría de medir las concentraciones de cada grupo de ingreso entre aquéllas. El modelo que aquí presentaremos nos informa sobre el primer componente de la segregación por grupos de ingreso.

\section{El modelo}

Hemos comentado que la segregación tiene dos determinantes: el conocimiento y el ingreso. Debido a que "segregación" es un concepto -útil para entender la distribución espacial de la homogeıeidad/heterogeneidad social, que designa el resultado del arrerlo de medios a fines en la disputa por el acceso a los recursos rbanos-sus determinantes son a la vez sus componentes, de maıra que si queremos precisarlo, tendremos que hacerlo a partir ie sus determinantes. En el modelo que vamos a presentar con datos de la ciudad de Tijuana, se toma en cuenta la segregación entre grupos sociales que agrupan individuos de acuerdo con sus ingresos. En una zona ocurre la segregación cuando hay homogeneidad en el ingreso de sus habitantes. Se considera que la segregación está determinada por el conocimiento y el ingreso, y a la 
vez está constituida por la predominancia en una zona de un grupo de ingreso sobre los demás.

Por el modo en que se presenta la información urbana desagregada del censo de población de 1990 , consideramos a cada área geoestadística básica (Ageb) como una zona de la ciudad, y a la población económicamente activa ocupada (PEAO) agrupada en cuatro rangos de ingreso: en el más bajo reciben menos de un salario mínimo (SM), les siguen los que reciben entre uno y dos SM, luego los que tienen más de dos y hasta cinco SM, y en el más alto rango están los que reciben más de cinco SM. Para controlar el cálculo debido a las variaciones del tamaño de la PEAO entre las Agebs, se tomaron las proporciones con que participan los rangos de ingreso en la PEAO de cada Ageb.

Escogimos un índice de diferenciación para medir la segregación, es decir el grado en que uno o algunos grupos de ingreso están concentrados en cada zona:

$$
G S_{j}=\sum_{i=1}^{n}\left(\frac{n}{n-1}\right)\left(P_{i j}-\frac{1}{n}\right)^{2}
$$

donde:

$G S_{j}=$ grado de segregación en la Ageb $j$

$\mathrm{P}_{\mathrm{ij}}=$ proporción en que el grupo de ingreso $i$ participa en la PEAO de la Ageb $j$

$\mathrm{n}=$ número de grupos de ingreso en la Ageb $j=4$

Este índice varía desde 0 hasta 1, de manera que si:

$G S_{\mathrm{j}}=0 \Rightarrow$ los grupos de ingreso se distribuyen de manera proporcional uniformemente en la Ageb $j$; gran heterogeneidad de la zona; nula segregación entre grupos

$G S_{j}=1=>$ un solo grupo de ingreso concentra a toda la PEAO de la Ageb j; hay total homogeneidad de la zona; total segregación entre grupos

Empíricamente, cada zona de la ciudad tiene un nivel de segregación que varía con el tamaño con que la delimitemos. El barrio conformado por una o dos calles tiene los mayores niveles de segregación, y a medida que incorporemos más área a la zona, la segregación disminuirá. Esto genera el problema de que las variaciones de la segregación, debidas a las características del método, no se pue- 
dan aislar de las variaciones producidas por los determinantes teóricos. La forma de reducir los errores resultantes consiste en tomar zonas de dimensión similar y que contengan la suficiente cantidad de población para poder estimar algún nivel de diversidad. La escala con que las Agebs se delimitaron quizá sea un poco grande debido a que los resultados del cálculo de la segregación tuvo un bajo promedio para el conjunto de las zonas. A pesar de ese bajo promedio general, se pudo obtener la suficiente variación estadística de la variable segregación para validar el modelo de regresión que la explica.

Para especificar el indicador de conocimiento consideramos que los conocimientos inconsciente y práctico (ya definidos) se pueden representar por variables censales que informen del tiempo de residencia en la ciudad de los pobladores de una Ageb. Del mismo modo, el conocimiento empírico se puede representar por variables del censo de población que informen de su grado de escolaridad y de la proporción de patrones de centros de trabajo (bajo el supuesto de que los patrones acceden a más instituciones y medios que los no patrones). Para construir índices que representen al conjunto de variables de conocimiento se aplicó el análisis factorial tomando como dato las proporciones de estas variables en la población total o PEAO en cada una de las 181 Agebs de la ciudad de Tijuana, cuyos resultados los vemos en el cuadro 1.

CUADRO 1

Resultados del análisis factorial del conocimiento

\begin{tabular}{|c|c|c|c|c|c|}
\hline \multicolumn{2}{|c|}{ Variable Communalidad $^{*}$} & \multirow{2}{*}{$\frac{\text { Factor }}{1}$} & \multirow{2}{*}{$\frac{\text { Eigenvalue }}{2.87786}$} & \multirow{2}{*}{ Pct of Var } & \multirow{2}{*}{$\begin{array}{c}\text { Cum Pet } \\
48.0\end{array}$} \\
\hline NACEBC & .94015 & & & & \\
\hline P5MRBC85 & .93690 & 2 & 1.76454 & & \\
\hline P15MCSEC & .23462 & & & & \\
\hline P15MCMBM & .87251 & & & & \\
\hline P18MCSUP & .93766 & & & & \\
\hline PPATRON & .72056 & & & & \\
\hline \multicolumn{6}{|c|}{$\begin{array}{c}\text { Factor } 1 \text { Factor } 2 \begin{array}{l}\text { Significado de variables } \\
\text { Porcentaje }\end{array} \\
\end{array}$} \\
\hline NACEBC & .01308 & .96953 & \multirow{3}{*}{\multicolumn{3}{|c|}{$\begin{array}{l}\text { Nacidos en Baja California } \\
\text { Población que residía en BC en } 1985 \\
\text { Población de } 15 \text { años y más con se- } \\
\text { cundaria completa }\end{array}$}} \\
\hline P5MRBC85 & .10764 & & & & \\
\hline P15MCSEC & -.44489 & .19155 & & & \\
\hline P15MCMBM & .90465 & .23264 & \multicolumn{3}{|c|}{$\begin{array}{l}\text { Población de } 15 \text { años y más con post- } \\
\text { media básica }\end{array}$} \\
\hline P18MCSUP & .95174 & .17847 & \multicolumn{3}{|c|}{$\begin{array}{l}\text { Población de } 18 \text { años y más con su- } \\
\text { perior }\end{array}$} \\
\hline PPATRON & .84780 & .04236 & \\
\hline
\end{tabular}

*Matriz de factores rotados: (correlación entre factores y variables). 
Tomando en cuenta las correlaciones de cada factor con las variables que los componen, consideramos que el factor 1 (F1) expresa el conocimiento empírico, y el factor 2 (F2), el conocimiento práctico, y sobre todo, el inconsciente. Con estos resultados calculamos los índices de ambos tipos de conocimiento en cada Ageb con la siguiente expresión:

$$
\begin{aligned}
I C F_{j}=\sum_{i=1}^{n} F P_{i} \frac{X_{i j}-X_{i}^{-}}{\delta_{i}} & \\
& \operatorname{con}:(j=1 \ldots m)
\end{aligned}
$$

donde:

$$
\begin{aligned}
& I C F \mathrm{n}_{j}=\text { índice de conocimiento de la Ageb } j \\
& \text { (ICF1 } 1_{j}=\text { empírico, } I C F 2_{i}=\text { inconsciente y práctico) } \\
& F P_{\mathrm{i}} \quad=\text { factor de ponderación de la variable } i \\
& \text { (correlación de la variable } i \text { con el factor } n \text { ) } \\
& X_{i j} \quad=\text { valor de la variable } i \text { en la Ageb } j \\
& X_{i} \quad=\text { promedio urbano de la variable } i \\
& \delta_{i}=\text { desviación estandar de la variable } i
\end{aligned}
$$

De esta manera especificamos al conjunto de las variables que entrarían en el análisis de determinación a través de una regresión múltiple. Las características de las variables especificadas se muestran en el cuadro 2.

Estos resultados nos dicen que el modelo es aceptable estadísticamente, y que la segregación está determinada en $63 \%$ por las variables que entraron en la ecuación (el método backward utilizado en la regresión no consideró en el modelo a la PEAO entre uno y dos SM por su baja correlación con la segregación y porque su varianza era contada por otras variables). Los valores "beta" son coeficientes de valores estandarizados; nos dicen la proporción en que la segregación se modifica ante el cambio de una unidad en la variable explicativa considerada, y que se puede esperar más segregación cuando se presenten bajos niveles, tanto en ambos tipos de conocimiento como en la proporción de PEAO más pobre, y cuando los salarios del rango medio al bajo sean los predominantes. Un resultado notable es que altos salarios no sean significativos en la determinación del nivel de segregación espacial. Esto podría explicarse por dos aspectos estadísticos y uno geo- 


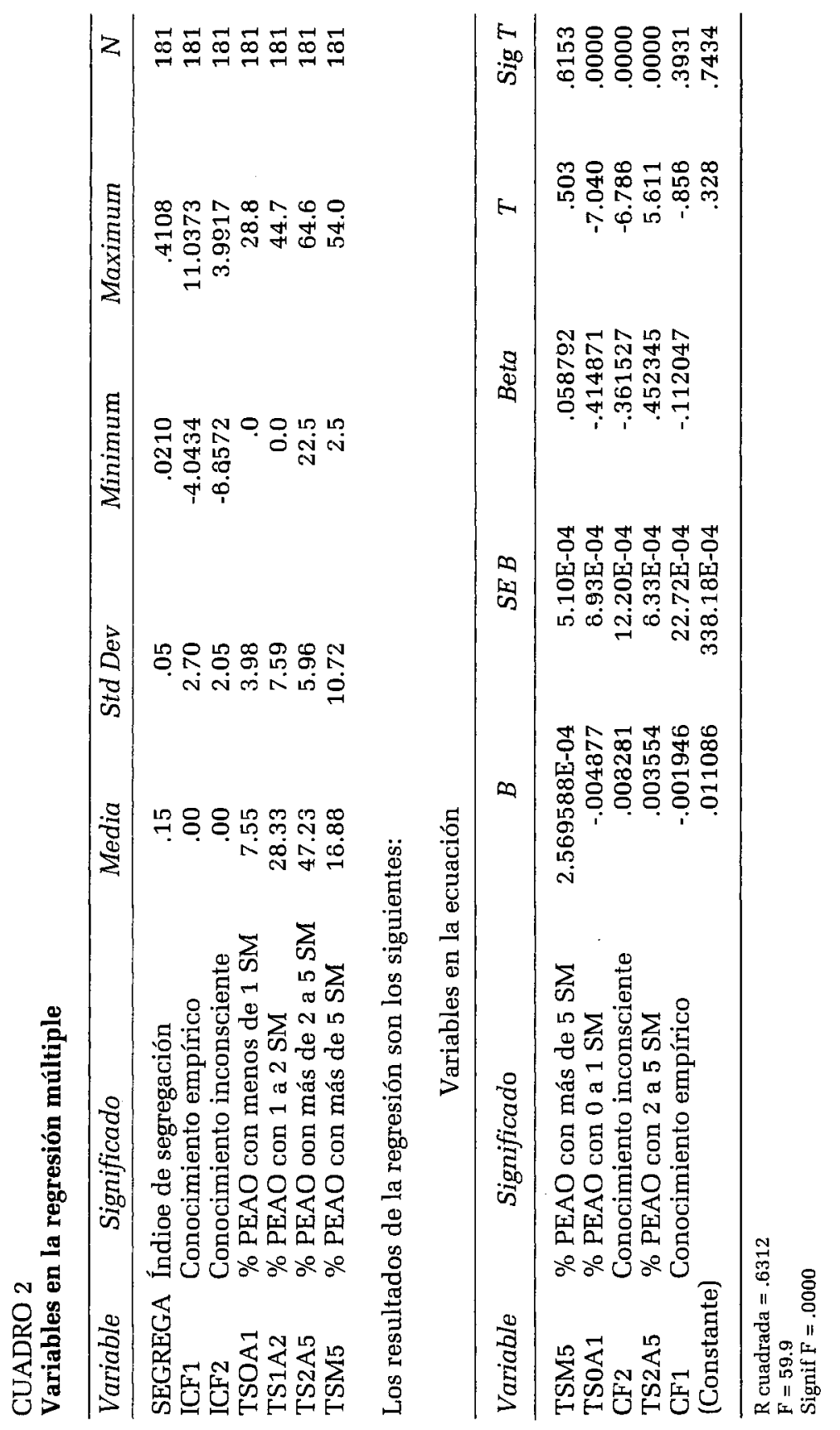


gráfico, a saber: i) la alta correlación que tiene esta variable con las dos de conocimiento, contadas las variaciones del conocimiento antes que la de altos salarios, para explicar la segregación; ii) que esa variable incluye a grupos de ingreso medio y alto; si hubiera manera de discriminar a éstos, quizá los altos ingresos estarían más estrechamente asociados con la segregación, y iii) las unidades geográficas de análisis, las Agebs, tienen una dimensión mayor a la conveniente, impidiendo variaciones mayores.

En síntesis, a pesar de su relativamente alto coeficiente de determinación, este modelo explicativo de la segregación espacial por ingreso es todavía exploratorio de los determinantes de la segregación. Debe ser considerado aún como una hipótesis de trabajo alrededor de la discusión conceptual expuesta a lo largo del escrito, la cual considera al conocimiento y a los ingresos como los principales determinantes del componente intrazonal de la segregación por el lado de la demanda de localización residencial. El lado de la oferta contaría la variación no explicada por este modelo, cuyos mejores indicadores debieran ser el precio del suelo y el tiempo de renovación de las edificaciones. Sin embargo, el propio indicador de segregación debiera mejorarse, de manera que tome en cuenta las variaciones entre zonas y no sólo las que ocurren dentro de cada zona como lo hace el indicador que aquí hemos presentado.

El modelo explica la segregación entre los grupos de ingreso, debida a la influencia del ingreso y del conocimiento; pero no mide la segregación entre los grupos de conocimiento. Explicar de manera conjunta ambas dimensiones de la segregación es una tarea complicada pero necesaria para la delimitación teórica y metodológica de la segregación como un concepto diferente al de localización.

\section{Bibliografía}

Alonso, William (1988), "A theory of the urban land Market", en R. Lake (ed.), Readings in urban analysis: perspectives on urban form and structure, New Jersey, Rutgers University, Center for Urban Policy Research.

Bourdieu, Pierre (1990), Sociología y cultura, México, Grijalbo y Conaculta.

Cadwallader, Martin (1985), Analytical urban geography, New Jersey, Englewood Cliffs, Prentice-Hall Inc.

Farley, John (1984), "P* Segregation Indices: What can they tell us about housing segregation in 1980?", en Urban Studies, vol. 21, núm. 3.

Germani, Gino (1988), "La ciudad como mecanismo integrador", en Bassols et al., Antología de sociología urbana, México, UNAM. 
Levy, John (1985), Urban and metropolitan economics, USA, McGrawHill Publishing Co.

Mills y Hamilton (1989), Urban Economics, United States, Scott, Foresman and Co.

Quijano, Aníbal (1988), "La formación de un universo marginal en las ciudades de América Latina”, en Bassols et al., Antología de sociología urbana, México, UNAM.

Smith, Richard (1991), "The mesurement of segregation change through integration and deconcentration, 1970-1980", en Urban Affairs, vol. 26, núm. 4.

Thrift, Nigel (1985), "Flies and germs: a geography of knowledge", en Gregory y Urry, Social relations and spatial structure, Nueva York, St. Martin's Press.

Wong, David (1993), "Spatial indices of segregation", en Urban Studies, vol. 30 , núm. 3. 